\title{
Detergent ringing true as a model for membranes
}

\section{J. Barber}

IT IS a frustrating business trying to understand how specific lipid species seem to optimize the functional properties of proteins that are embedded in the lipid bilayers that constitute most biological membranes. The problem is that membrane proteins are hydrophobic and therefore difficult to isolate and purify without using detergents to disrupt the membrane. Consequently, detergent molecules replace the lipid, and the best one can hope for is that information - for example, from high resolution $\mathrm{X}$-ray crystallography - on how the detergent is organized in relationship to the protein will be of some relevance to how the natural lipids are organized. Even then, the approach is hampered by the fact that detergent molecules will often not form ordered arrays because of thermal motion, particularly in their hydrocarbon tails. On page 659 of this issue', Roth et al. report an alternative approach, which makes use of neutron scattering.

Roth et al. have studied highly-ordered crystals of the reaction centre of the photosynthetic bacterium Rhodopseudomonas viridis, isolated with the detergent lauryl dimethyl amine oxide (LDAO). X-ray defraction of these crystals has shown that the reaction centre has a hydrophobic core consisting of eleven membrane-spanning $\alpha$-helices, five each from the L- and Msubunits of the protein and one from the H-subunit ${ }^{2}$. To be able to investigate the arrangement of detergent in the crystals, Roth et al. use the trick of varying the $\mathrm{H}_{2} \mathrm{O} / \mathrm{D}_{2} \mathrm{O}$ content of the crystals, recording variations of the phase of the structure factor as a fraction of contrast.

Unlike X-ray crystallography, this approach gives the low-resolution data 'needed to identify the domains formed by the detergent molecules in the crystals. Under optimal conditions, the authors observe the detergent as micellular rings around the transmembrane $\alpha$-helices of all three subunits. The thickness of the detergent rings along the $\alpha$-helices is 25-30 $\AA$, about twice the length of an LDAO molecule. Therefore, it seems that LDAO arranges itself around the hydrophobic core of the protein complex in the way depicted in text books for natural membrane lipids - as a bilayer.

Recently, Yeates et al. ${ }^{3}$ have conducted minimum energy calculations for the possible organization within a lipid bilayer of the reaction centre of $R$. sphaeroides, which has an atomic-resolution structure ${ }^{4}$ that is remarkably similar to that of the $R$. viridis reaction centre. Their analysis, however, predicts a thicker hydrophobic region for lipid-protein interactions of about $40-45 \AA$. The most likely explanation for this difference is that the bacterial reaction centre does not exist as a separate entity within the membrane but normally forms rart of a much larger complex involving the proteins that bind the lightharvesting bacteriochlorophylls 5 .

The neutron-scattering experiments of Roth et al. have also shown how the detergent domains interconnect throughout the crystal lattice. The results quite clearly show that LDAO not only interacts stereo-specifically with the hydrophobic region of the reaction centre but also creates optimal spacing for direct interactions to occur between the hydrophilic regions of adjacent proteins. Apparently, it is the interplay of these two factors that is vital for obtaining highly ordered lattices.

The message, therefore, is that there is no 'magic' detergent for crystallizing membrane proteins and each system must be explored independently. Moreover. it is likely that a similar message will apply to the packaging and organization of membrane proteins in their natural environment and that this underlies why nature has not been able to evolve a 'standard' lipid species for all systems.

J. Barber is Head of the Department of Biochemistry, Imperial College, London SW7 2AY, UK.

1. Roth, M., Lewit-Bentley, A., Michel. H.. Deisenhofer. $J$ Huber. R. \& Oesterhelt, D. Nature 340, 659-662 (1989)

Deisenhofer, J., Epp, O., Miki, K. Huber R. \& Michel. H. Nature 318, 618-624 (1985)

Yeates, T.O. Komiya. H. Rees, D.C. Allen, J.P. \& Feher G. Proc natn Acad Sci U S.A $84,6438-6442(1987)$ G. Proc. natn. Acad. Sci. U.S.A. 84, 6438-6442 (1987).
Allen, J.P. Feher, G., Yeates, T. O., Komiya, H. \& Rees D.C. Proc natn. Acad. Sci. U.S.A. 84, 6162-6166 (1987) 5. Stark, W. et al. EMBO J. 4, 777-783(1984).

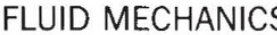 \\ Oil slicks still the waves}

\section{John C. Scott}

THE effect of oil in calming waves is not only one of the oldest phenomena in the scientific calendar', it is one which still excites much interest. A recent paper by Alpers and Huhnerfuss ${ }^{2}$ provides a timely summary of recent progress in understanding the calming effect of oil films on the sea surface. It also reports important progress in establishing the link between the processes of wind-energy input, waveenergy re-distribution and energy removal.

Contrary to popular belief not all slicks come from spilt crude oil. The ocean's natural productivity produces much harmless wave-damping material, and it is vital to distinguish the two causes. Concern over pollution, interest in the climatic influence of the sea surface, and the advent of powerful methods of observing the sea from space all ensure continuation of our interest in wave-damping effects.

The basic mechanism of wave damping was given by Horace Lamb, in the 1895 edition of his Hydrodynamics ${ }^{3}$, although the finer points still attract attention (and some controversy). Surface-active chemicals reduce the surface tension of water and the effect increases with the surface concentration of the chemical; when a wave stretches and compresses the surface it increases and decreases the surface tension, causing tangential boundary stresses which always oppose the wave motion. The film gives the surface a "dilational elasticity"; in the language of linear hydrodynamics the surface boundary condition changes from a 'free' surface to one which supports tangential stress, and a significant viscous boundary layer results. Note that the lost wave energy passes (as heat) into the water: it does not directly heat the film.

Inextensibility is the upper limit of elasticity, although real films never reach this. One of the most interesting effects of films is that the maximum damping can occur at quite small values of elasticity. Perhaps most remarkable is that the most effective films come from insoluble materials spread as single molecular layers; the effect scems quite disproportionate in this light.

A film usually gives a sharp damping peak, about twice that predicted for the inextensible case, and peaks appear in the variation of damping with wave frequency as well as with film elasticity. Alpers and Huhnerfuss prefer to consider these peaks as 'resonant' energy exchange between two wave modes - one the normal transverse surface waves and the other a highly damped longitudinal wave where motion is mainly to and fro at the horizontal surface. These latter waves are often called Marangoni waves, honouring the contribution to surface-film studies made by the nineteenth century Italian physicist. Although it is often found intuitively useful for considering the damping peaks. this division of the hydrodynamic solutions is not necessary ( $\mathrm{N}$. Thomas. personal communication). The conclusions reached by Alpers and Huhnerfuss are, however, not affected by this

One further interesting influence on wave damping is that the film need not be purely elastic. Viscoelasticity can arise from molecular factors in the film, and 